\section{On Low Complexity Robust Beamforming With Positive Semidefinite Constraints}

Chengwen Xing, Shaodan Ma, and Yik-Chung Wu

\begin{abstract}
This paper addresses the problem of robust beamforming for general-rank signal models with norm bounded uncertainties in the desired and received signal covariance matrices as well as positive semidefinite constraints on the covariance matrices. Two novel minimum variance robust beamformers are derived in closed-form. The first one basically is the closed-form version of an existing iterative algorithm, while the second one offers even better performance with respect to the first one. Both of them have the advantage of low complexity. The effectiveness and performance improvement of the proposed beamformers are verified by simulation results.
\end{abstract}

Index Terms-Robust beamforming, convex optimization, positive semidefinite constraint, minimax.

\section{INTRODUCTION}

Beamforming has long been an active research topic due to its ability to preserve desired signal and null interference. It has been widely utilized in radar, sonar, microphone array, wireless communications, and other fields [1]. Ideally, the beamformer is designed based on the true array responses to the received signal or the corresponding perfect covariance matrices. In practice, such actual/perfect information is difficult to obtain and the presumed/estimated array responses or covariance matrices are generally used for beamformer design. Unfortunately, mismatch between the actual and presumed/estimated information exists due to limited training or time varying environment, causing substantial performance degradation of beamformers. Robust beamforming, which takes the uncertainties of covariance matrices into account, therefore is of great importance in practical systems and has attracted a lot of research interest recently [2], [3].

For the simple point source signal model (i.e., rank-one model), a number of robust beamformers have been proposed in the literature [1]. However, in practice, the signal is usually incoherently scattered, resulting in a general-rank signal model, which calls for more sophisticated beamforming algorithms. In [5], a closed-form minimum variance (MV) robust beamformer was proposed for the general-rank signal model under norm bounded uncertainties in the desired signal and received signal covariance matrices. Unfortunately, in the formulation of [5], the positive semidefinite (PSD) constraints on the covariance matrices are not considered, which might result in over conservative solutions [2], [3]. Recently, with the additional PSD constraints considered, an iterative robust beamforming algorithm was proposed in [6]. While it provides better performance than the algorithm in [5], it has rather high computational complexity since a standard semidefinite programming (SDP) problem must be solved at each iteration by interior-point methods, which at least have polynomial complexity [8].

Manuscript received December 22, 2008; accepted May 19, 2009. First published June 23, 2009; current version published November 18, 2009. The work was supported in part by the General Research Fund, Project No. HKU 7181/07E and by the HKU Seed Funding Programme, Project No. 200711159056. The associate editor coordinating the review of this manuscript and approving it for publication was Prof. Andreas Jakobsson.

The authors are with the Department of Electrical and Electronic Engineering, The University of Hong Kong, Hong Kong (e-mail: cwxing@eee.hku.hk; sdma@eee.hku.hk; ycwu@eee.hku.hk).

Color versions of one or more of the figures in this paper are available online at http://ieeexplore.ieee.org.

Digital Object Identifier 10.1109/TSP.2009.2025823
In this paper, a general-rank signal model with uncertainties in the desired and received signal covariance matrices as well as the PSD constraints on the covariance matrices is considered. Two novel MV robust beamformers are derived in closed-form. The first one is a closed-form solution to the iterative beamforming algorithm in [6], thus offering the same performance as the iterative algorithm but with a much lower complexity. The second one provides even better performance compared to the first one since fewer approximations are made in the derivation. Simulation results confirm the effectiveness and performance improvement of the proposed beamformers. Note that both of the proposed beamformers have the advantage of low complexity due to the simple closed-form solutions and thus are attractive in practical systems.

The following notations are used throughout this paper. Boldface lowercase letters denote vectors, while boldface uppercase letters denote matrices. The notation $\mathbf{Z}^{\mathrm{H}}$ denotes the Hermitian of the matrix $\mathbf{Z}$, and $\mathbf{Z}^{*}$ is the conjugate of the matrix $\mathbf{Z}$. The symbol $\operatorname{Tr}(\mathbf{Z})$ denotes the trace of the matrix $\mathbf{Z}$.

\section{PROBLEM FORMULATION}

Sensor arrays are commonly used to detect signals and track targets. The array snapshot vector at the sensor array, which consists of $M$ sensors, can be expressed as

$$
\begin{aligned}
\mathbf{x}(k) & \triangleq\left[x_{0}(k) x_{1}(k) \cdots x_{M-1}(k)\right]^{\mathrm{T}} \\
& =\mathbf{s}(k)+\mathbf{i}(k)+\mathbf{n}(k)
\end{aligned}
$$

where $\mathbf{s}(k), \mathbf{i}(k)$ and $\mathbf{n}(k)$ are the desired signal, interference and noise components, respectively. Symbol $k$ is the time index. After narrowband beamforming, the output signal is $\omega^{\mathrm{H}} \mathbf{x}(k)$ where $\omega=\left[\begin{array}{llll}\omega_{0} & \omega_{1} & \cdots & \omega_{M-1}\end{array}\right]^{\mathrm{T}}$ is the complex weight vector. A simple but efficient algorithm to design the beamforming weight vector $\omega$ is the minimum variance (MV) beamforming, which is

$$
\min _{\omega} \omega^{\mathrm{H}} \mathbf{R} \omega \quad \text { s.t. } \omega^{\mathrm{H}} \mathbf{R}_{\mathbf{s}} \omega \geq 1
$$

where $\mathbf{R}_{\mathbf{s}}=\mathrm{E}\left\{\mathbf{s}(k) \mathbf{s}^{\mathrm{H}}(k)\right\}$ and $\mathbf{R}=\mathrm{E}\left\{\mathbf{x}(k) \mathbf{x}^{\mathrm{H}}(k)\right\}$ are the desired signal covariance matrix and signal-interference-plus-noise (SINR) covariance matrix, respectively. Notice that if the source is an incoherently scattered source, the rank of the matrix $\mathbf{R}_{\mathbf{s}}$ can be any integer between 1 and $M$. This is called the general rank signal model [1], [5]-[7]. If $\mathbf{R}$ and $\mathbf{R}_{s}$ are known exactly, the optimal solution can be shown to be [5]

$$
\omega_{\text {ideal }}=\mathcal{P}\left\{\mathbf{R}^{-1} \mathbf{R}_{\mathbf{s}}\right\}
$$

where $\mathcal{P}(\mathbf{Z})$ represents the principle eigenvector of the matrix $\mathbf{Z}$.

In practice, it is impossible to get the true values of $\mathbf{R}$ and $\mathbf{R}_{s}$. They are usually replaced by their estimated values and subject to errors. Therefore, we can write

$$
\begin{gathered}
\mathbf{R}=\hat{\mathbf{R}}+\boldsymbol{\Delta}_{2} \\
\mathbf{R}_{\mathbf{s}}=\hat{\mathbf{R}}_{\mathbf{s}}+\boldsymbol{\Delta}_{1}
\end{gathered}
$$

where $\hat{\mathbf{R}}_{\mathbf{s}}$ and $\hat{\mathbf{R}}$ are the estimates of $\mathbf{R}_{s}$ and $\mathbf{R}$, respectively [5], [6] and $\boldsymbol{\Delta}_{1}$ and $\boldsymbol{\Delta}_{2}$ are the estimation errors of $\mathbf{R}_{s}$ and $\mathbf{R}$, respectively.

Substituting (4) and (5) into (2) and introducing norm bounds on $\boldsymbol{\Delta}_{1}$ and $\boldsymbol{\Delta}_{2}$ [5], the optimization problem (2) becomes

$$
\begin{array}{cl}
\min _{\boldsymbol{\omega}} & \boldsymbol{\omega}^{\mathrm{H}}\left(\hat{\mathbf{R}}+\boldsymbol{\Delta}_{2}\right) \omega \\
\text { s.t. } & \boldsymbol{\omega}^{\mathrm{H}}\left(\hat{\mathbf{R}}_{\mathbf{s}}+\boldsymbol{\Delta}_{1}\right) \omega \geq 1 \\
& \left\|\boldsymbol{\Delta}_{2}\right\| \leq \gamma, \quad\left\|\boldsymbol{\Delta}_{1}\right\| \leq \varepsilon \\
\hat{\mathbf{R}}+\boldsymbol{\Delta}_{2} \succeq 0, \quad \hat{\mathbf{R}}_{\mathbf{s}}+\boldsymbol{\Delta}_{1} \succeq 0
\end{array}
$$


where $\|$.$\| is the Frobenius norm, \varepsilon$ and $\gamma$ are uncertianty norm bounds on $\boldsymbol{\Delta}_{1}$ and $\boldsymbol{\Delta}_{2}$, respectively and the last two inequalities specify that matrices $\hat{\mathbf{R}}_{\mathbf{s}}+\boldsymbol{\Delta}_{1}$ and $\hat{\mathbf{R}}+\boldsymbol{\Delta}_{2}$ are positive semidefinite (PSD).

Notice that (6) is a multicriterion optimization problem [8] that does not have an unique optimal solution, since the optimal solution to (6) depends on the unknown values of $\boldsymbol{\Delta}_{1}$ and $\boldsymbol{\Delta}_{2}$. In order to design the beamformer which is independent of $\boldsymbol{\Delta}_{1}$ and $\boldsymbol{\Delta}_{2}$, minmax approaches are frequently used

$$
\begin{array}{ll}
\min _{\boldsymbol{\omega}} & \max _{\boldsymbol{\Delta}_{2}} \omega^{\mathrm{H}}\left(\hat{\mathbf{R}}+\boldsymbol{\Delta}_{2}\right) \omega \\
\text { s.t. } & \min _{\boldsymbol{\Delta}_{1}} \omega^{\mathrm{H}}\left(\hat{\mathbf{R}}_{\mathbf{s}}+\boldsymbol{\Delta}_{1}\right) \omega \geq 1 \\
& \left\|\boldsymbol{\Delta}_{2}\right\| \leq \gamma \quad\left\|\boldsymbol{\Delta}_{1}\right\| \leq \varepsilon \\
& \hat{\mathbf{R}}+\boldsymbol{\Delta}_{2} \succeq 0, \quad \hat{\mathbf{R}}_{\mathbf{s}}+\boldsymbol{\Delta}_{1} \succeq 0 .
\end{array}
$$

When the last two PSD constraints are not considered, the worst case $\boldsymbol{\Delta}_{1}$ and $\boldsymbol{\Delta}_{2}$ occur on the boundaries of their respective uncertainty regions, and the closed-form solution was shown to be [5]:

$$
\boldsymbol{\omega}_{\mathrm{rob}}=\mathcal{P}\left\{(\hat{\mathbf{R}}+\gamma \mathbf{I})^{-1}\left(\hat{\mathbf{R}}_{\mathbf{s}}-\varepsilon \mathbf{I}\right)\right\}
$$

When the PSD constraints are considered, the worst case $\boldsymbol{\Delta}_{2}$ equals to $\gamma \omega \omega^{\mathrm{H}} /\|\omega\|^{2}[6]$, while the worst case $\boldsymbol{\Delta}_{1}$ cannot be calculated using conventional Lagrangian algorithm. Recently, in [6], by introducing $\boldsymbol{\Delta}$ (with norm bound $\eta$ ) as the uncertainty in the square root of $\mathbf{R}_{s}$ and with eigendecomposition $\hat{\mathbf{R}}_{\mathbf{s}}=\mathbf{Q}^{\mathrm{H}} \mathbf{Q}$, the PSD constraint $\hat{\mathbf{R}}_{s}+\boldsymbol{\Delta}_{1} \succeq$ 0 is replaced by $(\mathbf{Q}+\boldsymbol{\Delta})^{\mathrm{H}}(\mathbf{Q}+\boldsymbol{\Delta}) \succeq 0$ [6] and the optimization problem becomes

Pl:

$$
\begin{array}{ll}
\min _{\boldsymbol{\omega}} & \boldsymbol{\omega}^{\mathrm{H}}(\hat{\mathbf{R}}+\gamma \mathbf{I}) \boldsymbol{\omega} \\
\text { s.t. } & \min _{\boldsymbol{\Delta}}\left(\boldsymbol{\omega}^{\mathrm{H}}(\mathbf{Q}+\boldsymbol{\Delta})^{\mathrm{H}}(\mathbf{Q}+\boldsymbol{\Delta}) \boldsymbol{\omega}\right) \geq 1 \\
& \|\boldsymbol{\Delta}\| \leq \eta .
\end{array}
$$

Problem $P 1$ cannot be solved straightforwardly, because it is a nonconvex program. In [6], based on Cauchy-Schwarz inequality and Triangle inequality, and introducing $\mathbf{W}=\omega \omega^{\mathrm{H}}$, the optimization problem was further transformed into

$$
\begin{array}{ll}
\min _{\mathbf{W}} & \operatorname{Tr}((\hat{\mathbf{R}}+\gamma \mathbf{I}) \mathbf{W}) \\
\text { s.t. } & \operatorname{Tr}\left(\hat{\mathbf{R}}_{\mathbf{s}} \mathbf{W}\right)-\eta^{2} \operatorname{Tr}(\mathbf{W})-1 \geq 2 \eta \sqrt{\operatorname{Tr}(\mathbf{W})} \\
& \mathbf{W} \succeq 0, \quad \operatorname{rank}(\mathbf{W})=1 .
\end{array}
$$

The optimization problem (10) still cannot be solved easily because the term $2 \eta \sqrt{\operatorname{Tr}(\mathbf{W})}$ is nonconvex and the constraint $\operatorname{rank}(\mathbf{W})=1$ cannot be formulated as a linear matrix inequality (LMI). In [6], to avoid these two nonconvex constraints, an iterative algorithm based on Lagrangian relaxation has been proposed. More specifically, at the $k$ th iteration of the algorithm, the nonconvex term $2 \eta \sqrt{\operatorname{Tr}(\mathbf{W})}$ is replaced by the result of the previous iteration $2 \eta \sqrt{\operatorname{Tr}\left(\mathbf{W}_{k-1}\right)}$. At each iteration, the optimization problem becomes a standard SDP or LMI problem [8], which is

P2:

$$
\begin{array}{ll}
\min _{\mathbf{W}_{k}} & \operatorname{Tr}\left((\hat{\mathbf{R}}+\gamma \mathbf{I}) \mathbf{W}_{k}\right) \\
\text { s.t. } & \operatorname{Tr}\left(\left(\hat{\mathbf{R}}_{s}-\eta^{2} \mathbf{I}\right) \mathbf{W}_{k}\right) \geq c_{k} \\
& \mathbf{W}_{k} \succeq 0
\end{array}
$$

where $c_{k}=2 \eta \sqrt{\operatorname{Tr}\left(\mathbf{W}_{k-1}\right)}+1$. An obvious way to solve $P 2$ (when $k$ is fixed) is to use interior-point algorithms. In the following, we will show that closed-form solution exists for $P 2$, and iterative algorithm is not necessary.

\section{A CLOSED-FORM SOLUTION FOR P2}

At the $k$ th iteration, due to the PSD constraint on $\mathbf{W}_{k}$ in (13) and based on eigendecomposition, we can write

$$
\mathbf{W}_{k}=\mathbf{Q}_{\mathbf{W}_{k}} \mathbf{Q}_{\mathbf{W}_{k}}^{\mathrm{H}}
$$

where $\mathbf{Q}_{\mathbf{W}_{k}} \triangleq\left[\begin{array}{llll}\mathbf{q}_{1, k} & \mathbf{q}_{2, k} & \cdots & \mathbf{q}_{M, k}\end{array}\right]$ and $\mathbf{q}_{i, k}$ denotes the $i$ th column of the matrix $\mathbf{Q}_{\mathbf{W}_{k}}$. Substituting (14) into (11), we have

$$
\begin{aligned}
\operatorname{Tr}\left((\hat{\mathbf{R}}+\gamma \mathbf{I}) \mathbf{W}_{k}\right) & =\operatorname{Tr}\left((\hat{\mathbf{R}}+\gamma \mathbf{I}) \mathbf{Q}_{\mathbf{W}_{k}} \mathbf{Q}_{\mathbf{W}_{k}}^{\mathrm{H}}\right) \\
& =\operatorname{Tr}\left(\mathbf{Q}_{\mathbf{W}_{k}}^{\mathrm{H}}(\hat{\mathbf{R}}+\gamma \mathbf{I}) \mathbf{Q}_{\mathbf{W}_{k}}\right) \\
& =\sum_{i=1}^{M} \mathbf{q}_{i, k}^{\mathrm{H}}(\hat{\mathbf{R}}+\gamma \mathbf{I}) \mathbf{q}_{i, k} .
\end{aligned}
$$

Similarly, by substituting $\mathbf{W}_{k}=\mathbf{Q}_{\mathbf{W}_{k}} \mathbf{Q}_{\mathbf{W}_{k}}^{\mathrm{H}}$ into (12), the optimization problem $P 2$ can be reformulated as

$$
\begin{gathered}
\min _{\substack{\mathbf{q}_{i, k} \\
i=1,2, \ldots, M}} \sum_{i=1}^{M} \mathbf{q}_{i, k}^{\mathrm{H}}(\hat{\mathbf{R}}+\gamma \mathbf{I}) \mathbf{q}_{i, k} \\
\text { s.t. } \quad \sum_{i=1}^{M} \mathbf{q}_{i, k}^{\mathrm{H}}\left(\hat{\mathbf{R}}_{s}-\eta^{2} \mathbf{I}\right) \mathbf{q}_{i, k} \geq c_{k}
\end{gathered}
$$

which can be rewritten in a more compact form

$$
\min _{\mathbf{q}_{k}} \mathbf{q}_{k}^{\mathrm{H}} \tilde{\mathbf{R}} \mathbf{q}_{k} \text { s.t. } \mathbf{q}_{k}^{\mathrm{H}} \tilde{\mathbf{R}}_{s} \mathbf{q}_{k} \geq c_{k}
$$

where $\mathbf{q}_{k}=\left[\mathbf{q}_{1, k}^{\mathrm{T}} \mathbf{q}_{2, k}^{\mathrm{T}} \cdots \mathbf{q}_{M, k}^{\mathrm{T}}\right]^{\mathrm{T}}$ is the vector variable. The matrices $\tilde{\mathbf{R}}$ and $\tilde{\mathbf{R}}_{s}$ are defined as

$$
\begin{aligned}
\tilde{\mathbf{R}} & =\mathbf{I} \otimes(\hat{\mathbf{R}}+\gamma \mathbf{I}), \\
\tilde{\mathbf{R}}_{s} & =\mathbf{I} \otimes\left(\hat{\mathbf{R}}_{s}-\eta^{2} \mathbf{I}\right) .
\end{aligned}
$$

The optimization problem (17) is in the same form as the optimization problem (2). Based on a similar proof to that in [5], the solution is given by

$$
\begin{aligned}
\mathbf{q}_{k}^{\text {opt }} & =\mathcal{P}\left(\tilde{\mathbf{R}}^{-1} \tilde{\mathbf{R}}_{s}\right) \\
& =\mathcal{P}\left(\mathbf{I} \otimes(\hat{\mathbf{R}}+\gamma \mathbf{I})^{-1}\left(\hat{\mathbf{R}}_{s}-\eta^{2} \mathbf{I}\right)\right) \\
& =\mathbf{1} \otimes \mathcal{P}\left((\hat{\mathbf{R}}+\gamma \mathbf{I})^{-1}\left(\hat{\mathbf{R}}_{s}-\eta^{2} \mathbf{I}\right)\right)
\end{aligned}
$$

where $\mathbf{1}$ is all-one vector. The equality (21) is due to (18) and (19). The last equality is due to the property of Kronecker product [10]. From (22), the corresponding optimal column vectors $\mathbf{q}_{i, k}$ are

$$
\begin{aligned}
\mathbf{q}_{1, k}^{\mathrm{opt}} & =\mathbf{q}_{2, k}^{\mathrm{opt}}=\cdots=\mathbf{q}_{M, k}^{\mathrm{opt}} \\
& =\mathcal{P}\left((\hat{\mathbf{R}}+\gamma \mathbf{I})^{-1}\left(\hat{\mathbf{R}}_{s}-\eta^{2} \mathbf{I}\right)\right) .
\end{aligned}
$$

Since $\mathbf{W}_{k}=\mathbf{Q}_{\mathbf{W}_{k}} \mathbf{Q}_{\mathbf{W}_{k}}^{\mathrm{H}}=\sum_{i} \mathbf{q}_{i, k} \mathbf{q}_{i, k}^{\mathrm{H}}$, the optimal matrix $\mathbf{W}_{k}$ is

$$
\begin{aligned}
\mathbf{W}_{k}^{\text {opt }} \propto\left\{\mathcal { P } \left((\hat{\mathbf{R}}+\gamma \mathbf{I})^{-1}\right.\right. & \left.\left.\left(\hat{\mathbf{R}}_{s}-\eta^{2} \mathbf{I}\right)\right)\right\} \\
& \times\left\{\mathcal{P}\left((\hat{\mathbf{R}}+\gamma \mathbf{I})^{-1}\left(\hat{\mathbf{R}}_{s}-\eta^{2} \mathbf{I}\right)\right)\right\}^{\mathrm{H}} .
\end{aligned}
$$

Finally the optimal beamforming weight vector is

$$
\boldsymbol{\omega}_{P 2}^{\mathrm{opt}}=\mathcal{P}\left((\hat{\mathbf{R}}+\gamma \mathbf{I})^{-1}\left(\hat{\mathbf{R}}_{s}-\eta^{2} \mathbf{I}\right)\right) .
$$


Notice that the final closed-form solution is not a function of $c_{k}=$ $2 \eta \sqrt{\operatorname{Tr}\left(\mathbf{W}_{k-1}\right)}+1$. It means that the solution at the $k$ th iteration has no relation with the previous iteration. At each iteration, there is a closed-form solution and this solution is independent of the result of the previous iteration, so the iterative algorithm is not necessary and (25) is the closed-form solution for $P 2$.

It is worth mentioning that in addition to being the closed-form solution for $P 2,(25)$ automatically satisfies the rank one constraint in (10), which cannot be guaranteed by the iterative algorithm of [6]. Furthermore, in contrast to the closed-form solution of beamformer (25), the iterative algorithm in [6] only offers a solution of W. After obtaining $\mathbf{W}$, multiple Gaussian vectors with the covariance matrix $\mathbf{W}$ are generated and the one giving the largest signal-to-interference-plus-noise ratio (SINR) will be chosen [6]. This process incurs extra complexity with respect to the closed-solution (25).

It should be noticed that (25) is only the solution to $P 2$, which is a relaxed version of $P 1$ in (9). In the following, we will propose an approximated closed-form solution for the optimization problem $P 1$, which is closer to the original robust beamformer design problem under PSD constraints.

\section{PROPOSED SOLUTION FOR $P 1$}

In $P 1$, the worst case $\Delta$ can be obtained from the following minimization problem

$$
\begin{aligned}
\min _{\Delta} \omega^{\mathrm{H}} \Delta^{\mathrm{H}} \mathbf{Q} \omega+\omega^{\mathrm{H}} \mathbf{Q}^{\mathrm{H}} \Delta \omega & +\omega^{\mathrm{H}} \Delta^{\mathrm{H}} \Delta \omega \\
& +\omega^{\mathrm{H}} \mathbf{Q}^{\mathrm{H}} \mathbf{Q} \omega \text { s.t. }\|\Delta\| \leq \eta .
\end{aligned}
$$

This problem is a convex optimization problem with a quadratic objective function and its Lagrangian function is

$$
\begin{aligned}
\mathcal{L}=\boldsymbol{\omega}^{\mathrm{H}} \boldsymbol{\Delta}^{\mathrm{H}} \mathbf{Q} \boldsymbol{\omega}+\boldsymbol{\omega}^{\mathrm{H}} \mathbf{Q}^{\mathrm{H}} \boldsymbol{\Delta} \boldsymbol{\omega}+\boldsymbol{\omega}^{\mathrm{H}} \boldsymbol{\Delta}^{\mathrm{H}} \boldsymbol{\Delta} \boldsymbol{\omega} \\
+\boldsymbol{\omega}^{\mathrm{H}} \mathbf{Q}^{\mathrm{H}} \mathbf{Q} \boldsymbol{\omega}+\lambda\left(\operatorname{Tr}\left(\boldsymbol{\Delta} \boldsymbol{\Delta}^{\mathrm{H}}\right)-\eta^{2}\right)
\end{aligned}
$$

whose Karush-Kuhn-Tucker (KKT) conditions are [8]

1)

$$
\frac{\partial \mathcal{L}}{\partial \boldsymbol{\Delta}^{*}}=0
$$

2)

$$
\operatorname{Tr}\left(\boldsymbol{\Delta} \boldsymbol{\Delta}^{\mathrm{H}}\right)=\eta^{2}
$$

For convex optimization problems, KKT conditions are necessary and sufficient for the optimal solutions. Based on the first KKT condition, we have

$$
\frac{\partial \mathcal{L}}{\partial \boldsymbol{\Delta}^{*}}=\mathbf{Q} \omega \omega^{\mathrm{H}}+\boldsymbol{\Delta} \omega \omega^{\mathrm{H}}+\lambda \boldsymbol{\Delta}=0 .
$$

The solution of (28) is

$$
\tilde{\boldsymbol{\Delta}}=-\mathbf{Q} \omega \omega^{\mathrm{H}}\left(\lambda \mathbf{I}+\omega \omega^{\mathrm{H}}\right)^{-1} .
$$

Based on the matrix inversion lemma, the previous equation can be rewritten as

$$
\tilde{\Delta}=-\frac{\mathrm{Q} \omega \omega^{\mathrm{H}}}{\lambda+\omega^{\mathrm{H}} \omega}
$$

Substituting (30) into the second KKT condition, we have

$$
\frac{\operatorname{Tr}\left(\mathbf{Q} \boldsymbol{\omega} \boldsymbol{\omega}^{\mathrm{H}}\left(\mathbf{Q} \omega \omega^{\mathrm{H}}\right)^{\mathrm{H}}\right)}{\left(\lambda+\omega^{\mathrm{H}} \omega\right)^{2}}=\eta^{2} .
$$

From (30) and (31), the worst case $\tilde{\Delta}$ is

$$
\tilde{\Delta}=-\sqrt{\frac{\eta^{2}}{\omega^{\mathrm{H}} \mathbf{Q}^{\mathrm{H}} \mathbf{Q} \omega \omega^{\mathrm{H}} \omega}} \mathbf{Q} \omega \omega^{\mathrm{H}} .
$$

Substituting $\tilde{\Delta}$ into the objective function in (26), the first term $\omega^{\mathrm{H}} \boldsymbol{\Delta}^{\mathrm{H}} \mathbf{Q} \omega$ is given by

$$
\begin{aligned}
\omega^{\mathrm{H}} \tilde{\Delta}^{\mathrm{H}} \mathbf{Q} \omega & =-\omega^{\mathrm{H}} \mathbf{Q}^{\mathrm{H}} \omega^{\mathrm{H}} \omega \sqrt{\frac{\eta^{2}}{\omega^{\mathrm{H}} \mathbf{Q}^{\mathrm{H}} \mathbf{Q} \omega \omega^{\mathrm{H}} \omega}} \mathbf{Q} \omega \\
& =-\eta \sqrt{\frac{\omega^{\mathrm{H}} \mathbf{Q}^{\mathrm{H}} \mathbf{Q} \omega}{\omega^{\mathrm{H}} \boldsymbol{\omega}}} \omega^{\mathrm{H}} \omega .
\end{aligned}
$$

Similarly, the second and the third terms of the objective function in (26) are

$$
\begin{aligned}
& \omega^{\mathrm{H}} \mathbf{Q}^{\mathrm{H}} \tilde{\boldsymbol{\Delta}} \boldsymbol{\omega}=-\eta \sqrt{\frac{\boldsymbol{\omega}^{\mathrm{H}} \mathbf{Q}^{\mathrm{H}} \mathbf{Q} \boldsymbol{\omega}}{\omega^{\mathrm{H}} \boldsymbol{\omega}}} \boldsymbol{\omega}^{\mathrm{H}} \omega \\
& \omega^{\mathrm{H}} \tilde{\boldsymbol{\Delta}}^{\mathrm{H}} \tilde{\boldsymbol{\Delta}} \boldsymbol{\omega}=\eta^{2} \boldsymbol{\omega}^{\mathrm{H}} \omega .
\end{aligned}
$$

Based on (33), (34), and (35), the optimization problem $P 1$ becomes

$$
\begin{array}{ll}
\min _{\omega} & \boldsymbol{\omega}^{\mathrm{H}}(\hat{\mathbf{R}}+\gamma \mathbf{I}) \omega \\
\text { s.t. } & \boldsymbol{\omega}^{\mathrm{H}}\left(\hat{\mathbf{R}}_{\mathbf{s}}+\eta^{2} \mathbf{I}-2 \eta \sqrt{\frac{\omega^{\mathrm{H}} \hat{\mathbf{R}}_{\mathbf{s}} \omega}{\boldsymbol{\omega}^{\mathrm{H}} \omega} \mathbf{I}}\right) \omega \geq 1
\end{array}
$$

where we have used the definition $\hat{\mathbf{R}}_{s}=\mathbf{Q}^{\mathrm{H}} \mathbf{Q}$.

Unfortunately, in the constraint, the term inside the square root depends on the unknown $\omega$. This makes deriving the closed-form solution difficult. Here, based on the fact that $\omega^{\mathrm{H}} \hat{\mathbf{R}}_{\mathrm{s}} \omega \leq \lambda_{\max }\left(\hat{\mathbf{R}}_{\mathrm{s}}\right) \omega^{\mathrm{H}} \boldsymbol{\omega}$, we propose to replace $\sqrt{\left(\omega^{\mathrm{H}} \hat{\mathbf{R}}_{\mathrm{s}} \omega\right) /\left(\omega^{\mathrm{H}} \omega\right)}$ by its maximum value $\sqrt{\lambda_{\max }\left(\hat{\mathbf{R}}_{\mathbf{s}}\right)}$. The optimization problem (36) is relaxed as

$$
\begin{array}{ll}
\min _{\boldsymbol{\omega}} & \omega^{\mathrm{H}}(\hat{\mathbf{R}}+\gamma \mathbf{I}) \omega \\
\text { s.t. } & \boldsymbol{\omega}^{\mathrm{H}}\left(\hat{\mathbf{R}}_{\mathbf{s}}+\eta^{2} \mathbf{I}-2 \eta \sqrt{\lambda_{\max }\left(\hat{\mathbf{R}}_{\mathbf{s}}\right) \mathbf{I}}\right) \omega \geq 1 .
\end{array}
$$

Since

$$
\begin{aligned}
\omega^{\mathrm{H}}\left(\hat{\mathbf{R}}_{\mathbf{s}}+\eta^{2} \mathbf{I}-\right. & \left.2 \eta \sqrt{\frac{\omega^{\mathrm{H}} \hat{\mathbf{R}}_{\mathbf{s}} \omega}{\omega^{\mathrm{H}} \omega} \mathbf{I}}\right) \boldsymbol{\omega} \\
& \geq \omega^{\mathrm{H}}\left(\hat{\mathbf{R}}_{\mathbf{s}}+\eta^{2} \mathbf{I}-2 \eta \sqrt{\lambda_{\max }\left(\hat{\mathbf{R}}_{\mathbf{s}}\right) \mathbf{I}}\right) \boldsymbol{\omega},
\end{aligned}
$$

the new constraint actually corresponds to a smaller feasible set of $\boldsymbol{\omega}$ (i.e., a stricter constraint) than the original one. Noticing that (37) is also in the same form as (2), the optimal solution of (37) can be shown to be

$$
\boldsymbol{\omega}^{\mathrm{opt}}=\mathcal{P}\left\{(\hat{\mathbf{R}}+\gamma \mathbf{I})^{-1}\left(\hat{\mathbf{R}}_{\mathbf{s}}-2 \sqrt{\lambda_{\max }\left(\hat{\mathbf{R}}_{\mathbf{s}}\right)} \eta \mathbf{I}+\eta^{2} \mathbf{I}\right)\right\}
$$




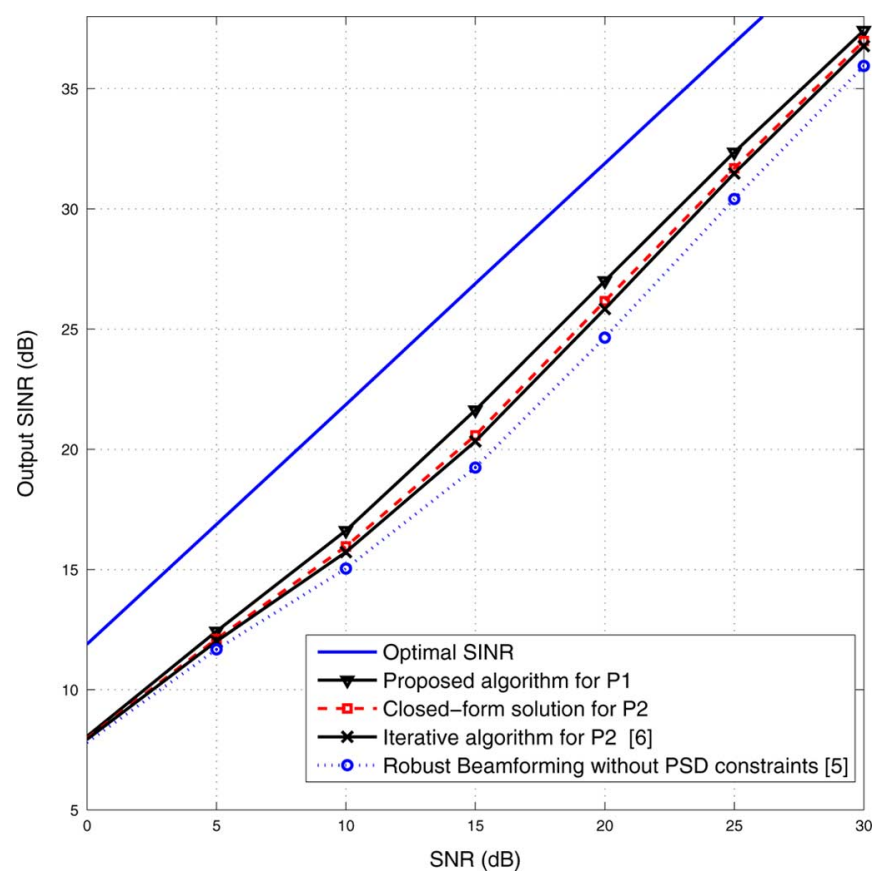

Fig. 1. SINR of output signal versus SNR for the robust beamforming without PSD constraints [5], iterative algorithm [6], the two proposed low complexity algorithms and the optimal SINR.

\section{Simulation Results AND Discussions}

A uniform linear array of $M=20$ omnidirectional sensors with adjacent sensors half a wavelength spaced apart is considered. The desired signal is assumed to be an incoherently scattered source with Gaussian angular power density whose central angle and angular spread equal to $30^{\circ}$ and $4^{\circ}$, respectively. The presumed angular power density of the signal is also Gaussian, but the presumed central angle and angular spread are $32^{\circ}$ and $6^{\circ}$, respectively. There is a single point-source interferer with interference-to-noise-ratio (INR) equals to $20 \mathrm{~dB}$. The interferer is modeled as a moving source with time varying direction-of-arrival (DOA) $\theta(k)=-30^{\circ}+10^{\circ} \sin \left(k / 15^{\circ}\right)$ [4]. The diagonal loading parameter is $\gamma=30$. The value $\varepsilon=9 \operatorname{Tr}\left(\mathbf{R}_{s}\right) / M$ is chosen as [5] and the value $\eta=0.75 \sqrt{\operatorname{Tr}\left(\mathbf{R}_{s}\right)}$ is chosen as [6]. In the simulations, an average of results from 500 trials is used to give each point in the figure.

Fig. 1 compares the output SINR of the two proposed algorithms, the iterative algorithm [6], the robust beamforming algorithm without PSD constraints [5], and the optimal SINR which corresponds to the beamformer based on perfect covariance matrices. For the iterative algorithm [6], 10 iterations are performed to design $\mathbf{W}$, and at each iteration, the Matlab software toolbox CVX [11] is used to solve $P 2$.

It can be seen that the proposed closed-form solution of $P 2$ has similar performance to the iterative algorithm in [6], since they are solving the same problem. Also they perform better than the algorithm without considering PSD constraints [5]. On the other hand, the proposed algorithm for $P 1$ performs better than both closed-form and iterative solutions of $P 2$, since $P 1$ is closer to the original beamformer design problem. At SNR $=15 \mathrm{~dB}$, the proposed algorithm for $P 1$ has $1 \mathrm{~dB}$ higher SINR output than the solutions of $P 2$.

Below, we compare the complexities of the two proposed algorithms to that of the iterative SDP algorithm in [6]. From (25) and (39), our two proposed algorithms need matrix inverse, matrix multiplication and eigendecomposition. For a $M \times M$ matrix, the complexities of these operations are all $O\left(M^{3}\right)$, so the total complexity is also $O\left(M^{3}\right)$. For Chen's algorithm in [6], it is an iterative algorithm and at each iteration, it involves solving a SDP problem. Primal-dual interior-point methods are usually used to solve SDP with the general complexity expression given in [9]. Applying the general expression to the problem in this paper. It can be shown that the complexity of Chen's algorithm is $O\left(M^{4.5} \ln (1 / \epsilon)\right)$, where $\epsilon$ is precision. For example, when $M=20$ and $\epsilon=0.01$, the reduction in complexities of the proposed algorithms, with respect to the iterative SDP in [6], is over 300 times.

\section{CONCLUSION}

In this paper, two novel algorithms were proposed for beamformer design under norm bounded uncertainties and PSD constraints on covariance matrices. The first one is the closed-form version of the iterative algorithm in [6]. The proposed closed-form solution basically has the same performance as the iterative algorithm but with a much lower complexity. The second algorithm proposed in this paper solves the beamformer design problem with fewer approximations, thus shows improved performance with respect to the first proposed algorithm.

\section{REFERENCES}

[1] J. Li and P. Stoica, Robust Adaptive Beamforming. Hoboken, NJ: Wiley , 2006.

[2] S. A. Kassam and H. V. Poor, "Robust techniques for signal processing: A survey," Proc. IEEE, vol. 73, no. 3, pp. 433-479, Mar. 1985.

[3] F. A. Dietrich, Robust Signal Processing for Wireless Communications, ser. Foundations in Signal Process., Commun., Netw., ISSN 1863-8538. Berlin, Germany: Springer, 2007.

[4] O. Besson and P. Stoica, "Decoupled estimation of DOA and angular spread for a spatially distributed source," IEEE Trans. Signal Process., vol. 48, pp. 1872-1882, Jul. 2000.

[5] S. Shahbazpanahi, A. B. Gershman, Z.-Q. Luo, and K. M. Wong, "Robust adaptive beamforming for general-rank signal models," IEEE Trans. Signal Process., vol. 51, pp. 2257-2269, Sept. 2003.

[6] H. H. Chen and A. B. Gershman, "Robust adpative beamforming for general-rank signal models using positive semidefinite covariance constraints," Proc. IEEE ICASSP, pp. 2341-2344, 2008.

[7] A. B. Gershman, C. F. Mecklenbruker, and J. F. Bohme, "Matrix fitting approach to direction of arrival estimation with imperfect spatial coherence of wavefronts," IEEE Trans. Signal Process., vol. 45, pp. 1894-1899, Jul. 1997.

[8] S. Boyd and L. Vandenberghe, Convex Optimization. Cambridge, U.K.: Cambridge Univ. Press, 2004.

[9] A. Ben-Tal and A. Nemirovski, Lectures on Modern Convex Optimization: Analysis, Algorithms, and Engineering Applications, ser. MPSSIAM Series on Optimiz.. Philadelphia, PA: SIAM, 2001.

[10] T. K. Moon and W. C. Stirling, Mathematical Methods and Algorithms for Signal Processing. Upper Saddle River, NJ: Prentice-Hall, 2000.

[11] M. Grant, S. Boyd, and Y. Y. Ye, CVX: Matlab Software for Disciplined Convex Programming [Online]. Available: http://www.stanford.edu/boyd/cvx/ Feb. 2007, available at: V.1.0RC3 